•研究报告・

\title{
库木塔格沙漠地区野骆驼活动节律与家域特征
}

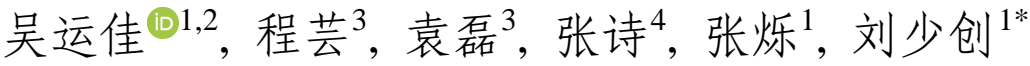

1. 中国科学院空天信息创新研究院, 北京 100094; 2. 中国科学院大学, 北京 100049; 3. 罗布泊野骆驼国家级自然保护区管理局, 乌鲁木齐 830011; 4. 中国地质大学(武汉)地理与信息工程学院, 武汉 430074

摘要: 野生双峰驼(Camelus ferus)生存于中亚沙漠腹地, 是国家I级重点保护野生动物。为探究野骆驼活动节律和家域状况, 了解其时间和空间尺度上的活动模式，为其有效保护管理提供支持。本研究于2012年5月至2013年7月利用GPS跟踪项圈先后 对库木塔格沙漠地区7峰野骆驼进行轨迹跟踪。利用跟踪数据对野骆驼活动节律进行分析, 并采用布朗桥模型对野骆驼家域 进行分析。结果表明: (1)野骆驼日活动节律呈现明显双峰模式, 属晨昏活动类型, 活动高峰期主要出现于上午6:00-9:00及下 午15:00-20:00。(2)野骆驼晨昏活动高峰存在明显的季节性变动, 双峰从暖季到冷季向中午移动, 按间隔时间长短排序为: 夏 季 $>$ 春季 $>$ 秋季 $>$ 冬季。(3)野骆驼日活动强度有明显的季节性差异, 大小关系为: 夏季 $>$ 秋季 $>$ 春季和冬季, 春季和 冬季间差异不显著。(4)野骆驼为核心家域利用类型，且存在多个核心家域，一些野骆驼家域分布于沙漠南北两侧，意味着其 具有横跨沙漠的运动能力。(5)野骆驼个体间家域面积差异显著, 性别间家域面积差异不显著。季节间家域面积差异显著, 从大 到小排序为: 夏季 $\left(1,256.27 \pm 427.45 \mathrm{~km}^{2}\right)>$ 春季 $\left(556.90 \pm 259.35 \mathrm{~km}^{2}\right)>$ 秋季 $\left(396.77 \pm 82.31 \mathrm{~km}^{2}\right)>$ 冬季 $\left(250.83 \pm 99.64 \mathrm{~km}^{2}\right)$ 。 关键词: 偶蹄类; 野骆驼; 活动节律; 家域; 布朗桥运动模型; 保护

吴运佳, 程芸, 袁否, 张诗, 张炼, 刘少创 (2021) 库木塔格沙漠地区野骆驼活动节律与家域特征. 生物多样性, 29, 1206-1214. doi: 10.17520/biods.2021104.

Wu YJ, Cheng Y, Yuan L, Zhang S, Zhang S, Liu SC (2021) Quantitative study on the activity rhythm and home range of wild camels (Camelus ferus) in the Kumtag Desert. Biodiversity Science, 29, 1206-1214. doi: 10.17520/biods.2021104.

\section{Quantitative study on the activity rhythm and home range of wild camels (Camelus ferus) in the Kumtag Desert}

Yunjia Wu ${ }^{\mathbb{1} 1,2}$, Yun Cheng 3 , Lei Yuan ${ }^{3}$, Shi Zhang ${ }^{4}$, Shuo Zhang ${ }^{1}$, Shaochuang Liu $^{1 *}$

1 Aerospace Information Research Institute, Chinese Academy of Sciences, Beijing 100094

2 University of Chinese Academy of Sciences, Beijing 100049

3 Xinjiang Lop Nor Wild Camel National Nature Reserve Administration, Urumqi 830011

4 School of Geography and Information Engineering, China University of Geosciences, Wuhan 430074

\section{ABSTRACT}

Aim: The wild camel (Camelus ferus) is a native species to the deserts of Central Asia. Further, it is considered a first class protected animal in China. This study aims to explore the daily activity rhythm and native ranges of wild camels both spatially and temporally, so as to provide data support for effective protection and management of wild camels in this region.

Methods: GPS tracking collars were used to gather movement data for seven wild camels in the Kumtag Desert from May 2012 to July 2013. This tracking data was then used to infer the daily activity rhythm of the wild camels. A Brownian bridge model was also applied to further investigate the native range of wild camels.

Results: The results of this study suggest that: (1) Daily activity rhythm data for these wild camels indicate a clear bimodal pattern, with peak activity periods occurring in the morning from 6:00 to 9:00 and in the afternoon from 15:00 to 20:00. This finding is consistent with the wild camels being a crepuscular species. (2) The double peak time of the wild camel's crepuscular activity is close to noon, occurring during the transition between the warm season and the cold season. The order of interval times between peaks compared seasonally is: summer $>$ spring $>$ autumn $>$ winter. (3) There are obvious seasonal differences in the daily activity intensity of wild camels. The relationships between daily 
activity intensity between seasons is: summer $>$ autumn $>$ spring and winter, where no clear difference was observed between spring and winter. (4) Wild camels belong to the core home range utilization type, often utilizing multiple core home ranges. The home ranges of the wild camels in this study exhibit a distribution between the north and south sides of the desert, suggesting that this species can move across the desert. (5) There are significant differences in home ranges among individuals, along with slight differences between sexes. Large variation in the home ranges of wild camels are observed seasonally, with the relative ordering from largest to smallest being: summer $(1,256.27 \pm$ $\left.427.45 \mathrm{~km}^{2}\right)>$ spring $\left(556.90 \pm 259.35 \mathrm{~km}^{2}\right)>$ autumn $\left(396.77 \pm 82.31 \mathrm{~km}^{2}\right)>$ winter $\left(250.83 \pm 99.64 \mathrm{~km}^{2}\right)$.

Key words: artiodactyla; wild camel; activity rhythm; home range; Brownian bridge movement model; conservation

日活动节律是指动物对昼夜环境条件周期性 变化的一种综合性适应, 主要受到温度、光照强度 等直接因素, 以及食物资源量、天敌数量等间接因 素的影响(尚玉昌, 2006)。它是动物行为学和生态学 的重要研究内容, 主要研究动物在不同时间、不同 季节条件下的活动强度及变化规律, 从而揭示动物 与环境相互作用信息及动物所采取的行为策略, 也 能反映动物个体的营养状态、社会地位以及生存压 力等参数(Halle \& Stenseth, 2000; 原宝东和孔繁繁, 2011)。家域指在一定时间内特定动物出现概率的区 域范围(Kernohan et al, 2001)。家域对于研究动物对 资源环境的适应与选择、种群密度以及社会关系等 生态学过程有着重要作用(张晋东等, 2013)。了解动 物活动节律及家域信息能够为其保护管理提供重 要参考依据(马建章等, 1999; 龙帅等, 2009; 袁磊等, 2015)。

野骆驼又名野生双峰驼(Camelus ferus), 被 IUCN红色名录列为极危物种(Hare, 2008), 也是国 家I级重点保护野生动物。野骆驼以其能适应极端荒 漠生存环境和饮用苦咸水的基因特性, 在世界生物 多样性保护中占有极为重要的地位, 也具有重要的 研究价值(Hare, 1997)。目前主要分布于中国阿尔金 山北麓(包括新疆和甘肃阿克塞安南坝)、塔克拉玛 干沙漠东部、罗布泊北部戛顺戈壁地区以及蒙古国 的中蒙边境外阿尔泰戈壁四个片块(Hare, 1997; 袁 国映等, 2012)。受气候变化和人类活动(如非法采 矿、非法狩猎)的影响, 野骆驼已经濒临灭绝。

野骆驼主要分布于中亚沙漠的无人区, 数量稀 少, 迁移能力强(Kaczensky et al, 2014), 调查研究 困难, 仅少数学者做了初步研究工作: (1)活动节律 研究。目前薛亚东等(2015)通过观察法对散养野骆 驼初冬活动时间分配及节律进行了初步研究, 但该 研究所观测野骆驼并非完全野生状态, 观测周期较 短, 且观察过程存在较高的人为干扰, 因此对野骆
驼活动节律需要进一步认识。与传统观察法相比, GPS跟踪项圈优势在于能够通过长时间自动跟踪获 得目标大量精确位置信息, 近年来已经成为重要的 野生动物研究技术(D'Eon \& Delparte, 2005)。根据 Ensing等(2014)的研究, 由GPS连续定位点所求得 的移动速率适合作为反映有蹄类动物日常活动强 度的指标, 因此, GPS跟踪项圈能够为野骆驼活动 节律监测提供支持。(2)家域研究。Kaczensky等 (2014)、袁否等(2015)、薛亚东等(2020)利用GPS跟 踪数据对野骆驼家域范围进行了研究, 研究算法均 采用最小凸多边形法(minimun convex polygons, $\mathrm{MCP}$ ), 但该方法易受异常点位影响, 同时会忽略 内部点位信息, 存在诸多弊端(张晋东等, 2013)。布 朗桥运动模型(Brownian bridge movement model, $\mathrm{BBMM}$ )克服了 MCP法的诸多不足, 有效地避免了 将活动斑块之间未被利用的区域误作为动物家域 的情况发生, 能更精确地估计家域范围(Horne et al, 2007)。针对上述情况, 为对野骆驼活动节律及家域 状况有更深入的认识, 支持野骆驼保护管理工作, 本文做了以下研究: (1)利用GPS跟踪项圈数据对野 骆驼日活动节律及节律季节性差异等进行研究。(2) 利用布朗桥运动模型对野骆驼家域空间分布、家域 利用方式、家域面积及家域季节性差异等进行研究。

\section{研究方法}

\section{1 研究区域}

研究区域为库木塔格沙漠及其周边地区 (图1), 范围: 89 $58^{\prime}-94^{\circ} 12^{\prime}$ E, $38^{\circ} 49^{\prime}-41^{\circ} 16^{\prime} \mathrm{N}$, 总面积达 $102,141 \mathrm{~km}^{2}$ 。位于甘肃省西部与新疆东南部交界处, 南临阿尔金山北麓, 北至阿奇克谷地, 西以罗布泊 为界, 同时包含西湖湿地及湿地东北部部分区域。 属温带大陆性气候, 极端干旱, 年均降水量不足 $50 \mathrm{~mm}$, 夏季气温常高达 $40^{\circ} \mathrm{C}$ 以上, 冬季最低气温 可达 $-28^{\circ} \mathrm{C}$, 多风沙, 气候条件恶劣。由于降水量极 


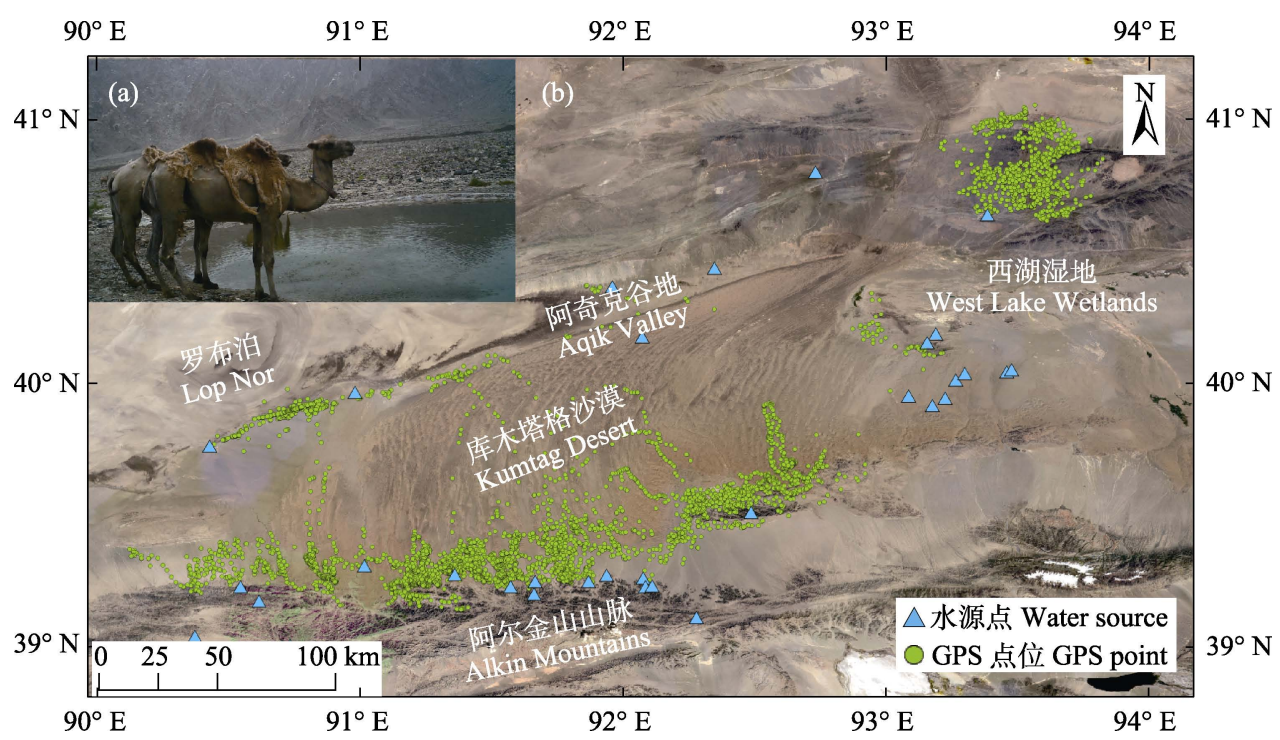

图1 野骆驼跟踪点位空间分布图。a: 佩戴GPS跟踪项圈的野骆驼; b: 2012年5月至2013年7月7峰野骆驼GPS定位位点、水源 点及所在区域卫星影像。

Fig. 1 Spatial distribution of tracking points of wild camels. a, Wild camels with GPS tracking collars; b, GPS positioning points of seven wild camels from May 2012 to July 2013, water sources and satellite image in the Kumtag Desert.

少，区域内长年无地表径流，沙漠内部存在一些常 年有水的盐泉, 野骆驼将其作为饮水点(萨根古丽 等, 2010)。植被覆盖较少, 主要分布于盐泉附近, 常 见植被主要以芦苇(Phragmites australis)、梭梭 (Haloxylon ammodendron)、多枝柽柳 (Tamarix ramosissima)、沙拐菄(Calligonum mongolicum)、泡 泡刺 (Nuraria sphaerocarpa)、合头草 (Sympegma regelii)、琵琶柴(Reaumuria soongorica)等荒漠植物 为主(萨根古丽等, 2010; 袁否等, 2015), 野骆驼首 选食用植物主要包括芦苇、梭梭、沙拐苯、泡泡刺 等(张莉和袁磊, 1997)。除野骆驼外, 该区域共存的 动物主要有藏野驴(Equus kiang)、我喉羚(Gazella subgutturosa)、岩羊(Pseudois nayaur)、盘羊(Ovis ammon)、狼(Canis lupus)、赤狐(Vulpes vulpes)等(袁 䂞等, 2015)。

\subsection{GPS项圈安置}

2012年5-10月, 在研究地区驾驶车辆寻找野骆 驼, 搜寻区域主要包括阿尔金山北麓、阿奇克谷地 以及西湖湿地北部一带。发现野骆驼后, 将其赶至 平坦区域, 车速维持在 20-30 km/h左右, 使野骆驼 位于车窗一侧, 由兽医使用麻醉枪麻醉野骆驼, 佩 戴可自动脱落的GPS跟踪项圈, 待野骆驼苏醒后原 地释放。最终共为 8 峰野骆驼成功佩戴项圈, 7 台设 备正常回传信号, 编号分别为C_NO1、C_NO2、
C_NO3、C_NO4、C_NO5、C_NO6、C_NO7，其中 4 峰为雌性, 3 峰为雄性。项圈每间隔一段时间通过 卫星通讯系统回传野骆驼位置信息, 包括跟踪目标 编码、经纬度坐标、定位日期及时间(UTC时间)等。 因项圈种类及初始设定存在差异, 编号为C_NO1的 项圈每隔24h回传1次位置信息, C_NO2回传信息间 隔从2-24 h不等, C_NO3、C_NO4、C_NO5、C_NO6、 C_NO7约2 h回传1次信息。这些项圈于2012年12月 至2013年7月先后停止工作(具体佩戴及脱落时间见 表1), 跟踪时间从163-366 d不等。经筛选后获得7 峰野骆驼的有效位置信息, 分别有299、797、1,958、 $3,364 、 2,128 、 2,448 、 2,658$ 个点位，点位空间分布 如图1b。

\section{3 野骆驼活动节律分析}

我们通过计算野骆驼 1 天内的连续运动速率来 反映其活动强度, 从而对日活动节律进行评估。对 每只野骆驼个体, 计算每对时间相邻定位点之间的 欧氏距离, 假设野骆驼在两个相邻点之间匀速运动, 将距离按单位小时离散化, 获得每单位小时内的移 动距离, 即速率 $(\mathrm{m} / \mathrm{h})$ 。由于跟踪期间野骆驼可能并 不进行直线运动, 这种方法产生了对移动速率的保 守估计。项圈回传定位点时间间隔存在差异, 较大 时间间隔不足以支持节律研究, 故去除了C_NO1与 C_NO2两个项圈的数据, 以定位时间间隔较小的 
表1 库木塔格沙漠地区7峰野骆驼家域及核心家域面积

Table 1 Home range and core area size of seven wild camels tracked in Kumtag Desert

\begin{tabular}{llllll}
\hline $\begin{array}{l}\text { 野骆驼 性别 } \\
\text { 编号ID Sex }\end{array}$ & $\begin{array}{l}\text { GPS项圈佩戴时间 } \\
\text { Start time of GPS trackers wearing }\end{array}$ & $\begin{array}{l}\text { GPS项圈脱落时间 } \\
\text { The time GPS trackers fell off GPS locations }\end{array}$ & $\begin{array}{c}\text { GPS点位数 } \\
\text { Home range }\left(\mathrm{km}^{2}\right)\end{array}$ & $\begin{array}{l}\text { 核心家域面积 } \\
\text { Core home range }\left(\mathrm{km}^{2}\right)\end{array}$ \\
\hline C_NO1 雌 Female & 2012.06 .01 & 2013.05 .31 & 299 & $2,476.38$ & 416.38 \\
C_NO2 雌 Female & 2012.05 .05 & 2012.12 .15 & 797 & $2,331.44$ & 309.00 \\
C_NO3 雄 Male & 2012.10 .13 & 2013.04 .01 & 1,958 & 481.05 & 45.97 \\
C_NO4 雌 Female & 2012.09 .21 & 2013.07 .02 & 3,364 & 929.55 & 56.45 \\
C_NO5 雄 Male & 2012.09 .29 & 2013.04 .19 & 2,128 & 528.08 & 47.11 \\
C_NO6 雄 Male & 2012.09 .19 & 2013.04 .25 & 2,448 & 806.73 & 117.52 \\
C_NO7 雌 Female & 2012.09 .22 & 2013.05 .16 & 2,658 & 212.28 & 27.30 \\
\hline
\end{tabular}

C_NO3、C_NO4、C_NO5、C_NO6、C_NO7五峰 野骆驼的位置信息作为节律研究数据。对每只野骆 驼个体, 计算跟踪期间各季节每单位小时内的平均 移动速率 $($ 根据当地实际气候状况, 季节划分方式 为: 春季(3-5月)、夏季(6-8月)、秋季(9-11月)、冬 季(12月至次年2月))。可视化后, 生成横轴为当地时 间 (UTC+6)、纵轴为速率(带标准误差)的野骆驼各季 节日移动速率折线图, 数据处理及制图使用 MATLAB R2020a软件完成。数据统计分析采用 SPSS 22.0软件, 对于野骆驼不同季节活动强度数 据, 经检验符合正态分布 (Kolmogorov-Smirnov检 验)后, 进行单因素方差分析(one-way ANOVA), 并 进行LSD两两比较。文中数值以平均值 \pm 标准误 (mean $\pm \mathrm{SE}$ ) 表示。

\section{4 野骆驼家域分析}

使用布朗桥运动模型(Horne et al, 2007)对7峰 野骆驼家域及核心家域进行计算, 家域采用95\%密 度估计表示, 核心家域采用50\%密度估计表示。家 域算法的实现基于 $\mathrm{R}$ 4.0.3 软件中 adehabitatLT、 adehabitatHR 以及 maptools 程序包(Calenge, 2006), 相关地图制作利用ArcGIS 10.2软件实现。数据统计 分析采用SPSS 22.0软件, 经检验符合正态分布后, 进行单因素方差分析或 $t$ 检验。季节划分及数值表示 方法与活动节律研究相同。

\section{2 研究结果}

\section{1 活动节律}

野骆驼在每个季节的活动节律均表现为双峰 模式(图2), 属于晨昏活动类型, 但在冬季晨昏活动 特征较其他季节存在一定差异, 表现为上午活动峰 值不明显, 且活动强度显著低于下午。野骆驼活动
高峰期主要出现在每日上午 6:00-9:00以及下午 15:00-20:00, 正午时分出现活动低谷。傍晚时分野 骆驼活动强度逐渐下降, 夜间活动强度达到最低, 第二天黎明活动强度又逐渐上升。整个跟踪期间, 其中1峰野骆驼最大日移动距离达 $91.7 \mathrm{~km}$ 。

野骆驼日活动节律模式在各个季节大体相同, 但同时存在明显的季节性变化。随季节变化, 两个 晨昏活动高峰距中午时间会发生改变, 按两峰间隔 时间由长到短排序依次为: 夏季 (6:00-7:00、 19:00-20:00)、春季(7:00-8:00、17:00-18:00)、秋季 (7:00-8:00、16:00-17:00)、冬季 (8:00-9:00、 15:00-16:00)。野骆驼在各个季节日均移动距离由大 到小排序为: 夏季 $(18.27 \pm 1.51 \mathrm{~km})$ 、秋季 $(11.80 \pm$ $0.66 \mathrm{~km}) 、$ 春季 $(8.20 \pm 0.52 \mathrm{~km}) 、$ 冬季 $(5.89 \pm 0.30 \mathrm{~km})$ 。 单因素方差分析表明, 野骆驼在不同季节活动强度 具有显著差异 $(F=24.42, d f=3, P<0.05)$ 。LSD两两 比较表明: 野骆驼夏季活动强度显著大于秋季, 秋 季活动强度显著大于春季和冬季, 春季和冬季之间 差异不显著 $(P>0.05)$ 。

\section{2 家域}

\subsection{1 家域空间分布}

跟踪的野骆驼虽为佩戴项圈后原地释放, 但部 分野骆驼项圈的佩戴地点并未包含在家域范围内 (图3)。从释放位置来看, C_NO1、C_NO2、C_NO3、 C_NO4、C_NO6释放位置均包含在家域范围内, 而 C_NO5与C_NO7则不然。对C_NO5与C_NO7前五 日的活动位点进行绘制(图3), 发现C_NO5释放后 先向北移动, 到达沙漠腹地后又过回至释放点东北 侧一冲积平原, 在该处生成家域范围, C_NO7释放 后直接向北移动穿越沙漠到达阿奇克谷地, 之后向 西移动到达罗布泊南部, 并在该区域生成家域范 

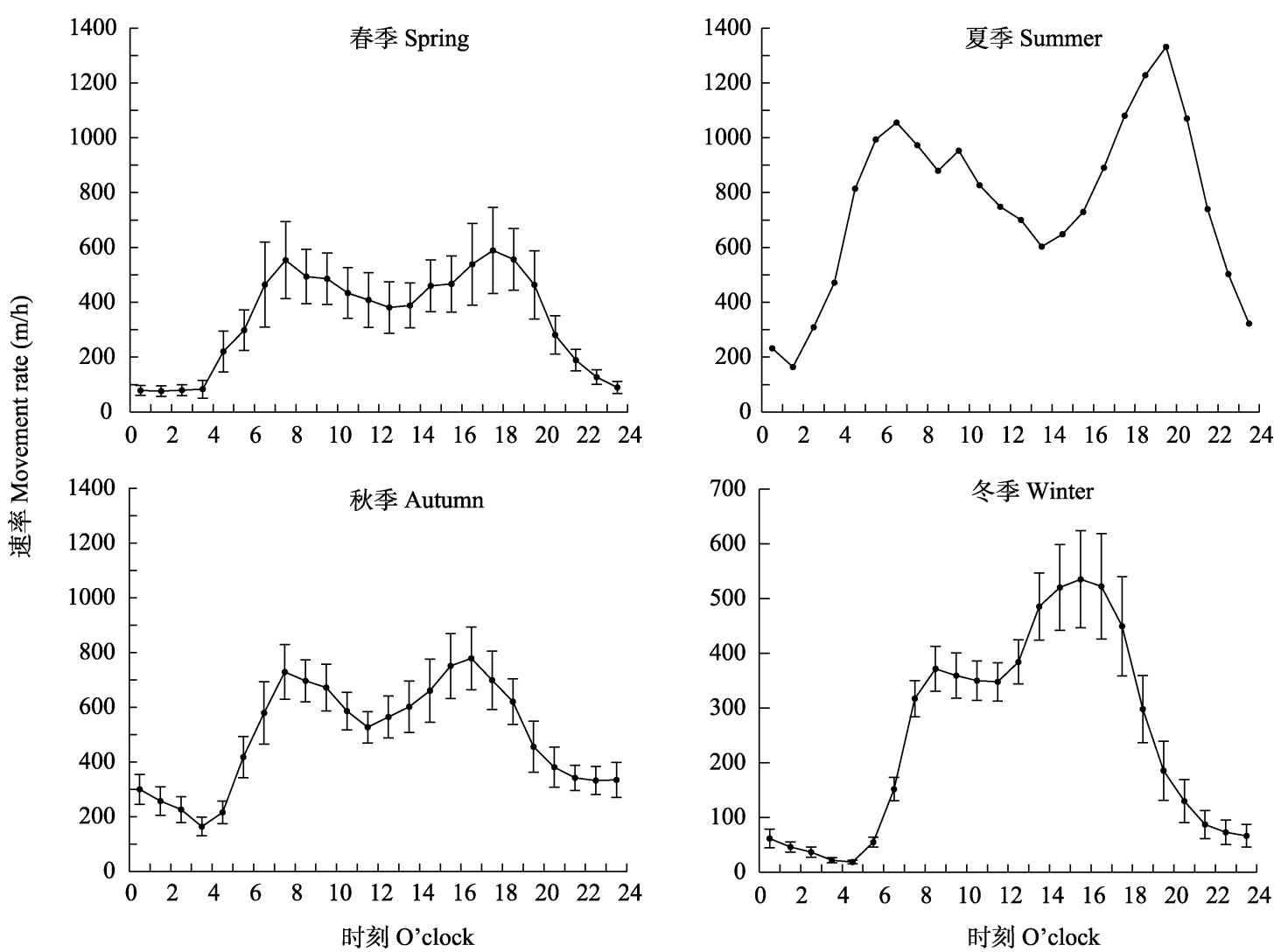

图2 春季 $(n=5)$ 、夏季 $(n=1)$ 、秋季 $(n=5)$ 、冬季 $(n=5)$ 野骆驼日活动节律。 $n$ 代表该季节参与计算的野骆驼数量。

Fig. 2 The daily activity patterns of wild camels in spring $(n=5)$, summer $(n=1)$, autumn $(n=5)$ and winter $(n=5) . n$ represents the number of wild camels involved in the calculation in the season.

围。抓捕行为可能使这 2 峰野骆驼产生了较强的应 激反应，离开了原有生存区域。

从家域空间分布来看, 野骆驼主要生存于以下 几个区域: 库木塔格沙漠南部与阿尔金山北麓交界 区域, 沙漠北部与罗布泊湖盆及阿奇克谷地交界的 干涸河床地带, 以及沙漠东部西湖湿地地区, 同时 还有 1 峰野骆驼在西湖湿地北部戈壁近圆形的封闭 区域内活动(图1b, 图3)。这些区域均集中在水源点 附近(图1b, 图3), 可为野骆驼提供充足的饮用水。 上述区域植被状况较好, 以梭梭、泡泡刺、沙拐杳 为主, 这些都是野骆驼的首选食用植物。由此可见, 野骆驼家域均选择了水资源和食物资源充足的生 境质量较好的地区。从核心家域来看, 野骆驼为多 核心家域利用方式, 每只个体均具有多个活动中心 (图3)。其中C_NO2在沙漠南部、北部、东部均存在 家域, C_NO3、C_NO7在沙漠南部及北部存在家域, 这意味着野骆驼具有南北向横跨沙漠的迁移能力。

\subsection{2 野骆驼家域面积}

利用2012年5月至2013年7月先后对7峰野骆驼
的跟踪数据, 计算得到每峰野骆驼的家域及核心家 域面积(表1)。7峰野骆驼平均家域面积(95\% BBMM) 为 $1,109.36 \pm 345.84 \mathrm{~km}^{2}$, 平均核心家域面积(50\% BBMM)为 $145.68 \pm 58.22 \mathrm{~km}^{2}$, 核心家域面积约占 家域面积的 $13.13 \%$ 。野骆驼个体间家域面积及核心 家域面积均有较大差异。其中C_NO1家域面积最大 $\left(2,476.38 \mathrm{~km}^{2}\right)$, C_NO7家域面积最小 $\left(212.28 \mathrm{~km}^{2}\right)$ 。 对于核心家域, 同样是C_NO1具有最大核心家域面 积(416.38 $\left.\mathrm{km}^{2}\right)$, C_NO7具有最小核心家域面积 $\left(27.30 \mathrm{~km}^{2}\right)$ 。雌性野骆驼家域面积 (1,487.41 \pm $\left.549.82 \mathrm{~km}^{2}\right)$ 与核心家域面积 $\left(202.28 \pm 95.36 \mathrm{~km}^{2}\right)$ 均 值分别大于雄性家域面积 $\left(605.29 \pm 101.63 \mathrm{~km}^{2}\right)$ 与 核心家域面积 $\left(70.00 \pm 23.66 \mathrm{~km}^{2}\right)$ 均值, 但独立样本 $t$ 检验表明, 家域与核心家域面积在性别间无显著差 异(家域: $t=1.578, d f=3.203, P=0.207>0.05$, 核 心家域: $t=1.344, d f=3.362, P=0.262>0.05)$ 。单因 素方差分析表明, 野骆驼季节间家域面积存在显著 差异 $(F=3.847, d f=3, P=0.025)$, 按家域面积均值 由大到小排序为: 夏季 $\left(1,256.27 \pm 427.45 \mathrm{~km}^{2}\right)>$ 春 


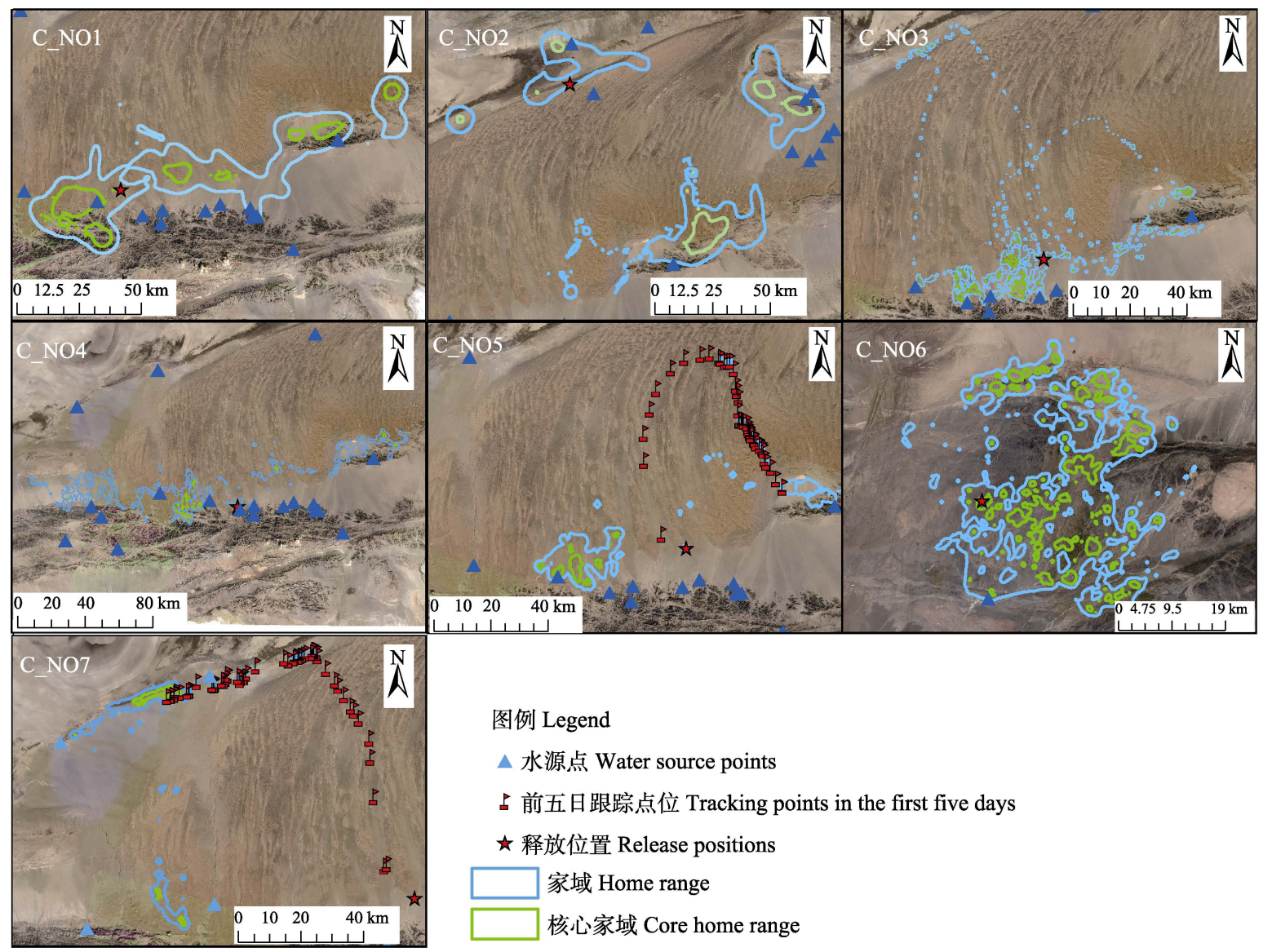

图3 库木塔格沙漠地区7峰野骆驼家域及核心家域分布

Fig. 3 Home ranges and core home ranges of seven wild camels in Kumtag Desert

表2 库木塔格沙漠地区7峰野骆驼季节性家域面积 $\left(\mathrm{km}^{2}\right)$

Table 2 Seasonal home range size of seven wild camels tracked in Kumtag Desert $\left(\mathrm{km}^{2}\right)$

\begin{tabular}{lllll}
\hline 野骆驼编号 ID & 春季 Spring & 夏季 Summer & 秋季 Autumn & 冬季 Winter \\
\hline C_NO1 & 865.33 & $1,506.37$ & 744.52 & 816.28 \\
C_NO2 & $1,930.1$ & $1,499.72$ & 563.74 & 161.91 \\
C_NO3 & 29.50 & - & 437.06 & 197.10 \\
C_NO4 & 264.10 & 762.71 & 308.26 & 56.12 \\
C_NO5 & 27.30 & - & 332.45 & 125.17 \\
C_NO6 & 659.95 & - & 339.36 & 316.99 \\
C_NO7 & 122.00 & - & 52.00 & 82.28 \\
均值 Mean & $556.90 \pm 259.35$ & $1,256.27 \pm 427.45$ & $396.77 \pm 82.31$ & $250.83 \pm 99.64$ \\
\hline
\end{tabular}

季 $\left(556.90 \pm 259.35 \mathrm{~km}^{2}\right)>$ 秋季 $(396.77 \pm 82.31$ $\left.\mathrm{km}^{2}\right)>$ 冬季 $\left(250.83 \pm 99.64 \mathrm{~km}^{2}\right)$ (表2)。

\section{3 讨论}

\section{1 野骆驼活动节律}

本研究为第一次利用GPS项圈对野生状态下野 骆驼活动模式进行量化分析, 结果表明, 库木塔格
沙漠地区野骆驼为晨昏活动类型，早晨和黄昏时刻 出现 2 个活动高峰。活动节律的晨昏模式在季节间 差异不显著, 但活动强度存在显著差异。这与其他 研究类似, 有蹄类动物普遍具有晨昏双峰活动模式 (Owen-Smith \& Goodall, 2014; 刘鹏等, 2019), 活动 强度在夏季达到高峰, 在冬季最低(Loe et al, 2007)。 野骆驼有很强的活动能力, 其中1只在跟踪期间 $24 \mathrm{~h}$ 
内移动距离高达 $91.7 \mathrm{~km}$ 。Kaczensky等(2014)对蒙古 国境内野骆驼跟踪中也发现野骆驼可在 $21 \mathrm{~h}$ 内移动 $74 \mathrm{~km}$ 。这种极强的活动能力便于野骆驼寻找食物 和水资源, 应对捕食者和恶劣天气条件的威胁。

本研究认为, 野骆驼活动模式主要受到环境温 度及食物资源的影响, 同时受到天敌、人类活动等 因素影响。野骆驼日活动节律模式有利于避开不同 季节不利气温条件的威胁, 冬季温度较低, 日最高 气温一般出现于下午14:00-15:00, 野骆驼在该季节 的日活动强度较低, 且上午活动强度显著低于下午 较温暖时刻, 由此可见, 环境温度较低时野骆驼会 降低活动意愿, 以减少热量消耗, 保存自身能量 (Moen, 1976)。有研究表明, 有蹄类动物在条件恶劣 的越冬条件下采用另一种生存策略, 即日出时在阳 坡晒太阳以维持体温, 从而降低自身代谢产热水平 (袁国映等, 2004; Signer et al, 2011), 这也可能是冬 季野骆驼活动量较低的原因。夏季野骆驼晨昏活动 高峰分别向更早和更晚方向移动, 这可能意味着野 骆驼要避开中午炎热时段, 在相对凉爽的时间活 动。MacArthur和Pianka (1966) 指出, 动物在食物资 源丰富时倾向于花费更多的时间寻找更优质的食 物, 在食物资源紧缺时则相反。库木塔格沙漠地区 夏季食物资源相对丰富, 野骆驼搜索采食多汁嫩草 和灌木, 而冬季草木枯黄, 食物质量差, 野骆驼搜 索优质食物意愿降低, 因此食物资源丰富程度也可 能是导致野骆驼夏季活动强度显著大于其他季节 的原因。野骆驼在库木塔格沙漠地区的主要天敌是 狼, 通常由四五只狼同时对野骆驼进行围攻(袁国 映等, 2004)。狼的捕食活动以清晨和黄昏最为频繁 (鞠弘等, 2010), 这两个时段也是野骆驼活动高峰期, 我们推测在野骆驼与狼长期共存的适应过程中, 为 了降低被捕食的风险而采取了晨昏活动方式。野骆 驼生性机警, 对人类有着极高的警惕性(Kaczensky et al, 2014), 晨昏活动也有利于避开白天人类活动 的影响。近年来我国对该区域生态环境保护力度不 断加强, 探矿、采矿等活动已全面停止, 但仍存在 放牧活动及非法探险、旅游、穿越活动(周永祥, 2020)。为方便资源运输, 近年来在沙漠周边区域修 建有西气东输管道、铁路、高速公路及砂石道路(高 丽君等, 2020), 公路产生的噪声会对野骆驼产生干 扰, 影响其活动节律, 同时这些因素也会造成栖息 地破碎化, 对野骆驼迁移及受食产生影响。

\section{2 野骆驼家域}

袁否等(2015)采用MCP法得出8峰野骆驼家域 面积为3,826-11,724 km², 薛亚东等(2020)采用同样 方法得到3峰野骆驼的家域面积为1,162-8,152 km 上述研究与本文用BBMM法的结果 $\left(212-2,476 \mathrm{~km}^{2}\right)$ 均表明, 不同野骆驼个体间家域面积存在较大差 异。动物个体间家域面积差异较为常见, 也见于普 氏野马(Equus przewalskii)、羚 (Budorcas taxicolor) 等的研究中(官天培等, 2015; 王渊等, 2016)。这与 动物年龄、性别、食物资源、捕食风险以及跟踪时 间等因素有关(龙博等, 2011)。本研究虽与袁磊等 (2015)研究采用同组实验数据, 但家域面积范围远 小于其结果, 也远小于薛亚东等(2020)的结果, 究 其原因主要有以下两点: 其一, 最小凸多边形算法 受异常值影响较大, 个别边缘GPS位点决定了动物 家域面积的大小(张晋东等, 2013)。野骆驼存在多个 核心家域，当其在各个核心家域之间迁移时，该方 法将迁移路线周围大片未涉足区域划分到家域范 围内, 因此采用MCP法会对结果造成高估, 并不能 准确反映野骆驼实际利用家域面积。BBMM法是一 种概率模型, 既考虑了每个活动中心, 又考虑到了 从一个区域向另一个区域移动的活动路径, 并将定 位点时间间隔纳入计算, 避免了点位之间的空间自 相关性(张晋东等, 2013), 比传统的MCP法更能准 确估计野骆驼实际家域范围。其二, 可能是由监测 时间差异引起，本研究数据采集时间与袁磊等 (2015)研究一致(2012-2013年), 早于薛亚东等(2020) 的2014-2016年, 近年来国家加强了野骆驼分布区 内的生态环境保护, 增设野骆驼饮水点, 对采矿、 探矿、旅游探险等人为活动进行限制。可能因各项 生态保护政策的逐步落实, 野骆驼生境破碎化程度降 低, 迁移廊道得到恢复, 野骆驼适宜分布区域扩大。

本研究表明野骆驼家域面积在季节间差异显 著, 家域面积由大到小为: 夏季 > 春季 > 秋季 > 冬季, 而袁否等(2015)得出野骆驼各季节平均家域 面积由大到小为: 秋季 > 冬季 > 夏季 > 春季, 薛亚东等(2020)得出的结论为春季大于冬季, 秋季 和夏季面积最小。二人对于季节性家域面积研究均 采用MCP法, 对于研究结果的不一致, 同样考虑是 由算法差异或样本量较小等因素引起。野骆驼季节 性家域面积差异体现了其对环境变化的适应, 有蹄 类动物家域面积季节性变化主要与采食、饮水需求, 
繁殖状态以及环境温度等因素有关(龙博等, 2011; 王渊等, 2016)。夏季库木塔格沙漠地区降雨充沛, 形成大量水泡供野骆驼饮用, 且植物含水量较高, 该时期野骆驼对水源点依赖程度较低(薛亚东, 2014), 饮水需求对夏季家域扩大的驱动作用较小。 依据前文野骆驼季节间活动节律分析结果, 为获取 优质食物资源, 野骆驼夏季具有最高活动强度, 因 此采食需求可能是夏季家域面积扩大的主要驱动 力。春秋两季家域面积大小与活动强度结果相反。 根据薛亚东等(2020)的研究, 每年春季为野骆驼发 情繁殖期, 野骆驼可能为了寻找伴侣而大范围移动, 产生较多的迁移行为, 导致产生较大家域范围。秋 季野骆驼活动强度虽然大于春季, 但活动范围可能 较春季相对稳定, 迁移行为较少, 家域忠诚度高, 家域范围较小。冬季气候寒冷, 食物资源少, 野骆 驼为减少自身热量消耗而降低活动强度, 同时, 冬 季降雪为野骆驼提供了水源, 对水源点依赖小, 最 终导致了冬季较小的家域范围。

综上, 本文利用GPS跟踪数据对野骆驼活动节 律及家域进行了分析, 在未来的野骆驼保护工作中, 我们应当根据野骆驼活动节律及家域分布, 尽量避 免保护区内道路、管线等基础设施修建活动对野骆 驼的干扰, 根据家域范围合理调整保护区边界。加 强对野骆驼分布区内非法旅游、非法穿越、放牧等 活动的管制力度。对于野骆驼家域核心区域可进行 重点保护, 如增设饮水点, 进行植被养护等。关于 库木塔格沙漠地区野骆驼还有很多问题亟待深入 研究, 如不同性别不同年龄间野骆驼活动节律差 异、节律影响因素的检验、野骆驼生境适宜度制图、 野骆驼种群数量和空间分布调查、未来气候变化对 野骆驼生境适宜度的影响等, 这对于野骆驼保护管 理具有重要意义。

\section{ORCID}

吴运佳 (D) https://orcid.org/0000-0003-0770-3094

\section{参考文献}

Calenge C (2006) The package “adehabitat” for the R software: A tool for the analysis of space and habitat use by animals. Ecological Modelling, 197, 516-519.

D’Eon RG, Delparte D (2005) Effects of radio-collar position and orientation on GPS radio-collar performance, and the implications of PDOP in data screening. Journal of Applied Ecology, 42, 383-388.
Ensing EP, Ciuti S, de Wijs FALM, Lentferink DH, Hoedt A, Boyce MS, Hut RA (2014) GPS based daily activity patterns in European red deer and North American elk (Cervus elaphus): Indication for a weak circadian clock in ungulates. PLoS ONE, 9, e106997.

Gao LJ, Xie ZJ, Guli SG, Baheti TLWH (2020) The main distribution area and population change of wild camel in Lop Nur. Environmental Protection of Xinjiang, 42(1), 36-41. (in Chinese with English abstract) [高丽君, 谢正君, 萨根古丽, 特列吾汗.巴合提 (2020) 罗布泊野骆驼主要 分布区域及种群变化. 新疆环境保护, 42(1), 36-41.]

Guan TP, Ge BM, Chen LM, You ZQ, Tang ZH, Liu H, Song YL (2015) Home range and fidelity of Sichuan takin. Acta Ecologica Sinica, 35, 1862-1868. (in Chinese with English abstract) [官天培, 葛宝明, 谌利民, 游章强, 唐中海, 刘 昊, 宋延龄 (2015) 四川羚牛的家域与忠诚度. 生态学 报, 35, 1862-1868.]

Guli SG, Shala, Yuan L (2010) Inhabit environment and adaptive character of wild camel in Wild Camel National Nature Reserve of Lop Nor in Xinjiang. Environmental Protection of Xinjiang, 32(2), 30-33. (in Chinese with English abstract) [萨根古丽, 沙拉, 袁䂞 (2010) 罗布泊 野骆驼国家级自然保护区野骆驼的栖息环境及适应特征. 新疆环境保护, 32(2), 30-33.]

Halle S, Stenseth NC (2000) Activity Patterns in Small Mammals: An Ecological Approach. Springer-Verlag, New York.

Hare J (1997) The wild Bactrian camel Camelus bactrianus ferus in China: The need for urgent action. Oryx, 31, 45-48.

Hare J (2008) Camelus ferus. The IUCN Red List of Threatened Species 2008: e.T63543A12689285. https://dx. doi.org/10.2305/IUCN.UK.2008.RLTS.T63543A12689285. en. (accessed on 2020-01-25)

Horne JS, Garton EO, Krone SM, Lewis JS (2007) Analyzing animal movements using Brownian bridges. Ecology, 88, 2354-2363.

Ju H, Zhang YB, Liu BW (2010) Research on the conflicts between wolf and herdsman in Dalai Lake Natural Reserve of Inner Mongolia. Modern Agricultural Science and Technology, (17), 322-323, 329. (in Chinese with English abstract) [鞠弘, 张云冰, 刘丙万 (2010) 内蒙古达㐘湖保 护区狼与牧民冲突研究. 现代农业科技, (17), 322-323, 329.]

Kaczensky P, Adiya Y, von Wehrden H, Mijiddorj B, Walzer C, Güthlin D, Enkhbileg D, Reading RP (2014) Space and habitat use by wild Bactrian camels in the Transaltai Gobi of southern Mongolia. Biological Conservation, 169, 311-318.

Kernohan BJ, Gitzen RA, Millspaugh JJ (2001) Analysis of animal space use and movements. In: Radio Tracking \& Animal Populations (eds Millspaugh JJ, Marzluff JM), pp. 125-166. Academic Press, Cambridge.

Liu P, Liu ZS, Gao H, Li ZZ, Zhang ZR, Teng LW (2019) Comparing the activity pattern of red deer (Cervus alashanicus) and blue sheep (Pseudois nayaur) using 
camera-traps in Helan Mountains. Acta Ecologica Sinica, 39, 9365-9372. (in Chinese with English abstract) [刘鹏, 刘振生, 高惠, 李宗智, 张致荣, 滕丽微 (2019) 基于红 外相机技术的贺兰山同域分布阿拉善马鹿和岩羊活动规 律研究. 生态学报, 39, 9365-9372.]

Loe LE, Bonenfant C, Mysterud A, Severinsen T, Øritsland NA, Langvatn R, Stien A, Irvine RJ, Stenseth NC (2007) Activity pattern of arctic reindeer in a predator-free environment: No need to keep a daily rhythm. Oecologia, 152, 617-624.

Long B, Chen Z, Li YC (2011) Effects of different factors on animal home range size. Journal of Hainan Normal University (Natural Science), 24, 439-443. (in Chinese with English abstract) [龙博, 陈忠, 李玉春 (2011) 不同因素 对动物家域面积的影响. 海南师范大学学报(自然科学 版), 24, 439-443.]

Long S, Zhou CQ, Wang WK, Pan L, Hu JC (2009) Diurnal behavioral rhythm, time budgets and group behavior of dwarf blue sheep in summer. Zoological Research, 30, 687-693. (in Chinese with English abstract) [龙帅, 周材权, 王维奎, 潘立, 胡锦真 (2009) 矮岩羊夏季活动节律、时 间分配和集群行为. 动物学研究, 30, 687-693.]

Ma JZ, Xu L, Zhang HH, Bao XK (1999) Activity rhythms of sables (Martes zibellina) in the Daxing'anling of China. Acta Theriologica Sinica, 19, 95-100. (in Chinese) [马建章, 徐利, 张洪海, 包新康 (1999) 大兴安岭地区紫貂的活动 节律. 兽类学报, 19, 95-100.]

MacArthur RH, Pianka ER (1966) On optimal use of a patchy environment. The American Naturalist, 100, 603-609.

Moen AN (1976) Energy conservation by white-tailed deer in the winter. Ecology, 57, 192-198.

Owen-Smith N, Goodall V (2014) Coping with savanna seasonality: Comparative daily activity patterns of African ungulates as revealed by GPS telemetry. Journal of Zoology, 293, 181-191.

Shang YC (2006) Behavioral rhythms of animals. Bulletin of Biology, 41(10), 8-10. (in Chinese) [尚玉昌 (2006) 动物 的行为节律. 生物学通报, 41(10), 8-10.]

Signer C, Ruf T, Arnold W (2011) Hypometabolism and basking: The strategies of alpine ibex to endure harsh over-wintering conditions. Functional Ecology, 25, 537-547.

Wang Y, Chu HJ, Han LL, Tao YS, Bu L, Liu Z, Jiang ZG (2016) Factors affecting the home range of reintroduced Equus przewalskii in the Mt. Kalamaili Ungulate Nature Reserve. Acta Ecologica Sinica, 36, 545-553. (in Chinese with English abstract) [王渊, 初红军, 韩丽丽, 陶永善, 布 兰, 刘钊, 蒋志刚 (2016) 野放普氏野马 (Equus przewalskii)家域面积及其影响因素. 生态学报, 36, 545-553.]

Xue YD (2014) Activity Pattern and Habitat Analysis of Wild Bactrian Camel (Camelus ferus) in Kumtag Desert. PhD dissertation, Chinese Academy of Forestry, Beijing. (in Chinese with English abstract) [薛亚东 (2014) 基于红外
相机的库木塔格沙漠地区野骆驼活动规律和适宜生境研 究. 博士学位论文, 中国林业科学研究院, 北京.]

Xue YD, Li DQ, Li J (2020) Habitat selection and migration pattern of wild Bactrian camel (Camelus ferus) in the Kumtag Desert, China based on satellite tracking and positioning technology. Scientia Silvae Sinicae, 56, 192-198. (in Chinese with English abstract) [薛亚东, 李迪 强, 李佳 (2020) 基于卫星追踪定位技术的库木塔格沙 漠野骆驼生境利用和迁移规律. 林业科学, 56, 192-198.]

Xue YD, Sun ZC, Wu P, Zhang YG, Li DQ (2015) Time budgets and diurnal activity rhythms of re-introduced wild Bactrian camels in semi-free environment during early winter. Scientia Silvae Sinicae, 51, 169-174. (in Chinese with English abstract) [薛亚东, 孙志成, 吴鹏, 张于光, 李 迪强 (2015) 散养条件下野生双峰驼初冬活动时间分配 及节律. 林业科学, 51, 169-174.]

Yuan BD, Kong FF (2011) Research on mammal activity rhythm. Journal of Anhui Agricultural Sciences, 39, 1056-1058, 1162. (in Chinese with English abstract) [原宝 东, 孔繁繁 (2011) 哺乳动物活动节律研究进展. 安徽农 业科学, 39, 1056-1058, 1162.]

Yuan GY, Yuan L, Shihati MMLH (2004) Wild Bactrian camels: 'tramps' in desert gobi. Life World, 31(5), 24-33. (in Chinese) [袁国映, 袁否, 马木利汉·术哈提 (2004) 野 双峰驼: 沙漠戈壁里的 “流浪汉”. 生命世界, 31(5), 24-33.]

Yuan GY, Zhang Y, Yuan L (2012) Lop Nur Nature Reserve. Science Press, Beijing. (in Chinese) [袁国映, 张宇, 袁否 (2012) 罗布泊自然保护区. 科学出版社, 北京.]

Yuan L, Ma H, Cheng Y, Yang H, Guli SG, Adiya Y, Zhu HY, Ma YQ, Wang JL, Zhang S, Ma XY, Diao QA, Liu SC (2015) Qualitative study of wild camels (Camelus ferus) home range in Lop Nur, China. Biodiversity Science, 23, 314-320. (in Chinese with English abstract) [袁否, 马浩, 程芸, 杨欢, 萨根古丽, Adiya Y, 朱海涌, 马友青, 王建 林, 张炼, 马杏叶, 刀庆安, 刘少创 (2015) 罗布泊野骆 驼的家域特征及其意义. 生物多样性, 23, 314-320.]

Zhang JD, Hull V, Ouyang ZY (2013) A review of home range studies. Acta Ecologica Sinica, 33, 3269-3279. (in Chinese with English abstract) [张晋东, Hull V, 欧阳志云 (2013) 家域研究进展. 生态学报, 33, 3269-3279.]

Zhang L, Yuan L (1997) Analysis of feeding habits of wild Bactrian camel in different distribution areas of the world. Environmental Protection of Xinjiang, 20(3), 60-64. (in Chinese) [张莉, 袁砧 (1997) 世界野双峰驼各分布区食 性分析. 新疆环境保护, 20(3), 60-64.]

Zhou YX (2020) Analysis of the current status of wild camel (Camelus bactrianus) in Annanba area and study of a species protection strategy. Chinese Journal of Wildlife, 41, 814-817. (in Chinese with English abstract) [周永祥 (2020) 安南坝片区野骆驼生存现状分析及保护策略. 野生动物 学报, 41, 814-817.]

(责任编委: 蒋志刚 责任编辑: 问文杰) 